\title{
Experimental Study on Non-English-teacher Parents' Participation in Family English Education for Young Children*
}

\author{
Xiuning Yang \\ Science and Technology College Gannan Normal \\ University \\ Ganzhou, China 341000
}

\author{
Pingsheng Zeng \\ Science and Technology College Gannan Normal \\ University \\ Ganzhou, China 341000
}

\begin{abstract}
Today, as English education is getting younger and younger, the issue of English education for young children in which parents of non-English-teacher participate is worth exploring. Experiments show that most non-English-teacher parents are willing and able to participate in family English education for young children, but they are still in a state of underdevelopment. Therefore, we need to explore the principles, content, and patterns of parental involvement to provide them with scientific guidance.
\end{abstract}

Keywords-non-English-teacher parents; participation; young children; family English education

\section{INTRODUCTION}

In line with the global education strategy, foreign language education has received unprecedented attention in countries around the world. At the Paris International Language Conference in 1989, the participating scholars put forward the idea of starting foreign language education from young children. Since then, foreign language education has become a trend of younger age in the world and has become a relatively independent research field. In 2001, the Ministry of Education promulgated the "Notice on Actively Promoting the Opening of English in Primary Schools" and decided to gradually open English classes from the third grade from the beginning of the fall. Since then, children's English education in China has developed rapidly. Nowadays, all sectors of society and parents attach great importance to the English education of children (especially young children), and the number of parents who asked to begin to teach English in kindergartens and in the lower grades of primary schools has increased, and various English training institutions have emerged. In line with this, many scholars in China began to explore the theoretical and practical basis for English education for young children. However, because English education for young children is still a brand-new subject, from the existing research, the perspectives are mostly on micro-level education and teaching, mainly related to curriculum, goals, teaching

*[Fund Project] "Investigation and Analysis of Parenting Concepts of Elderly Maternal Families under the Background of Comprehensive TwoChild Education", sponsored by the Jiangxi University humanities research project in 2015 (Project No. JY1505). materials, teachers, students, teaching and so on. As for the discussion of parents' participation in English education for young children, there is less concern, mainly the summary of experience of some English teachers. Undoubtedly, English education for young children is inseparable from family factors. Effective parental involvement can stimulate children's interest in English learning, improve children's English learning, and is conducive to discuss scientific English education models for young children. Then, how non-English-teacher parents participate in family English education for young children is an important and urgent issue at the moment.

\section{ASSUMPTIONS, GOALS, OBJECTS AND TIME OF THE EXPERIMENT}

Experiment assumptions: Most non-English-teacher parents are willing and able to participate in family English education for young children; effective parental involvement can stimulate low-school children's interest in English learning and improve their learning; non-English-teacher parents' participation in young children's family English education has its inherent regularity, and the way in which different categories of non-English-teacher parents participate in English education for young children should be different; appropriate guidance is conducive to improving the enthusiasm and effectiveness of parental participation.

Experiment target: The first is to explore the possibility of non-English-teacher parents participating in family English education for young children; the second is to analyze the principles and contents of non-English-teacher parents participating in family English education for young children; the third is to explore the effective mode of nonEnglish-teacher parents participating in family English education for young children; the fourth is to study the organization and guiding strategies of non-English-teacher parents participating in family English education for young children.

Experiment subjects: On the basis of careful analysis of the questionnaire, the research team selected 12 families as experiment subjects for the convenience of research. The experiment subjects were divided into experimental group 
(Wu Jiayi, Yang Mohan, Wang Yanqi, Lai Yuchun, Wang Xuhang, Liu Ziyi) and control group (Qiu Fengyuan, Song Yuqing, Zhang Zenan, Zhong Bowen, Wang Peiyi, Wang Han) and both of them involves 6 families. Specifically: the experimental group includes Wu Jiayi (female, second grade, has participated in the training, father takes the responsibility, Chinese teacher, undergraduate), Yang Mohan (female, second grade, did not participate in the training, father takes the responsibility, undergraduate), Wang Yanqi (male, first Grade, has participated in the training, mother takes the responsibility, college degree), Lai Yuchun (male, first grade, did not participate in the training, mother takes the responsibility, college degree), Wang Xuhang (male, senior grade of kindergarten, has participated in the training, mother takes the responsibility, technical school graduate), Liu Ziyi (female, senior grade of kindergarten, did not participate in the training, mother takes the responsibility, junior middle school graduates), and the control group includes Qiu Fengyuan (male, second grade, has participated in the training, mother takes the responsibility, mathematics teacher, undergraduate), Song Yuqing (female, second grade, did not participate in the training, father takes the responsibility, undergraduate), Zhang Zenan (male, first Grade, has participated in the training, mother takes the responsibility, technical school graduate), Zhong Bowen (male, first grade, did not participate in the training, mother takes the responsibility, technical school graduate), Wang Peiyi (female, senior grade of kindergarten, has participated in the training, mother takes the responsibility, high school graduate), Wang Han (female, senior grade of kindergarten, did not participate in the training, mother takes the responsibility, junior middle school graduates). Selection principle: one-to-one correspondence, as consistent as possible. The questionnaires of the parents in the experimental group and the control group were basically the same. However, there is a big difference between the $\mathrm{Wu}$ Jiayi family and the corresponding Qiu Fengyuan family, of who the gender of both the children and the parents is different. The experimental instructors are mainly Yang Xiuning, Liu Tingting and others.

Experiment time: June 2015 to August 2017.

\section{EXPERIMENT PROCEDURE}

\section{A. Developing an Experiment Plan (June 2015 to August 2015)}

Under the communication with the members of the research group, the parents of the experimental group and the teachers of the relevant training institutions, the experimental plan is formulated, which mainly includes the time and place of the experiment, the content of the experiment, the mode of experiment, the control of the experiment and the feedback of the evaluation of the experiment.

\section{B. Holding an Experiment Mobilization and Training Session (September 2015)}

Organize team members, experimental group parents and relevant training institutions to hold mobilization and training sessions: to raise parents' awareness of participating in experiments, to help parents build confidence, to clarify plans for parents to participate in experiments, to tell parents the requirement to participate in experiments, to teach parents the skills to participate in experiments, etc.

\section{Implementation of Organizing the Experiment (September 2015 to June 2017)}

The first stage: September 2015 to January 2016: the experimental group family chooses the training institution independently, and the parents are guided to purchase the books about family education (for self-study). According to the teaching materials and learning tasks of the training institutions, uses the Fun dubbing to improve the participation effectiveness of the parents (listening, speaking, and reciting). Experimental teachers and training institutions should be well trained in participating skills. The members of the research team should communicate with the parents once a week, hold a discussion meeting at the end of each month, and hold an experience exchange and feedback meeting at the end of the semester.

The second stage: February 2016 to August 2016: All the experimental group families continued the first stage of the experiment, and let the parents of Wu Jiayi and Lai Yuchun, who have good oral English, conduct daily conversations with their children. At the end of each month, a symposium was held, and at the end of the semester, an experience exchange and feedback meeting was held. By comparing the experimental group with the control group, the experimental teacher drew an experimental summary.

The third stage: September 2016 to June 2017: The control group was changed into an experimental group, and the same experiment was conducted according to the experimental contents and methods of the first and second stages. At the same time, the guidance and monitoring of the original experimental group was stopped. After the experiment, compare the original experimental group with itself, compare the current experimental group (the original control group) with itself, and compare the current experimental group with the original experimental group to form an experimental summary.

The fourth stage: July 2017 to August 2017: Organize an experimental summary meeting, analyze the experimental results, and form an experimental report.

\section{EXPERIMENT EFFECT}

In the past two years, through the joint efforts of all members of the research team, the experiment has achieved relatively satisfactory results, mainly as follows:

\section{A. Deepening Parents' Understanding of Participation}

Before the experiment, most parents thought that "participation" was "to accompany, to provide learning materials or tools". After the first stage of the experiment, the parents of the experimental group believed that "participation" could be "the formulation of the learning plan, the adjustment of the learning process, the evaluation of the learning effect, and the discussion of the learning"; After the 
second stage of the experiment, the two parents with good oral English in the experimental group thought that "participation" can also be "companion of learning together, comprehensive display learning process (such as recording learning audio, video)", etc. That is, turn passive into active, happy to participate, and happy to learn. In contrast, the family in the control group did not change much about the participation, but in the third stage of the experiment, when the original control group became the experimental group, the parents of this group also had similar changes to the original experimental group. In other words, parents' understanding of "participation" can be deepened through experiments.

\section{B. Improving Parents' Participation Ability Faster}

Before the experiment, most of the parents' participation was passive, and they did not know how to participate, mainly to carry out low-level activities such as letting children participate in interest classes and helping children buy school supplies. After three stages of experiment, most parents can develop participation plans according to specific situations, can communicate in a multi-directional way for children's learning, can actively learn and improve their participation ability, can actively share the joyful participation, and parents with better oral level can learn to become children's learning partners. That is to say, under the guidance of experimental teachers and teachers of training institutions, parents' ability to participate has been greatly improved, and parents are fully capable of participating in children's English education.

\section{Inspiring Children's Interest in Learning}

Before the experiment, most children were very willing to learn English, but their "willingness" often only stayed at "curious, fresh" rather than interest itself. After three stages of experiment, children spend more time learning English at home, and the frequency is increased, the way (listening, speaking and watching) is increased, and their learning is active and happy. In other words, children's learning interests are stimulated by the effective participation of parents.

\section{Significantly Enhancing the Learning Effect of Children}

Before the experiment, most children only completed the listening, reciting and simple writing tasks of the teachers of the training institutions. After three stages of experimentation, most children can actively, happily listen, follow, imitate, dare to talk daily, and actively display. Moreover, the degree of difficulty they can accept is much higher than before, and the quality is greatly improved. In other words, the participation of parents has improved the effect of children learning English.

\section{E. Variance Analysis of the Experimental Conditions (Ignoring Differences in Children's Gender in Learning)}

The first stage of the experiment: the overall performance of the parents in the experimental group was better than that of the control group parents. Among the 6 families in the experimental group: after the guidance, they did not find that the 6 parents had significant differences in their understanding of participation, but the participation ability of Liu Ziyi was relatively weak; The learning interest of these 6 children was not significantly different, and no significant difference was found in the learning effect.

The second stage of the experiment: There were some differences in the 6 families in the experimental group: the participation attitude and participation skills of both $\mathrm{Wu}$ Jiayi's and Lai Yuchun's parents with better oral English are better than those of other families. From the implementation of the participation plan, the parents of $\mathrm{Wu}$ Jiayi, Yang Mohan, Wang Yanqi and Lai Yuchun, who have higher academic qualifications, are obviously better than the parents of Wang Xuhang and Liu Ziyi; The learning initiative and learning effect of Wu Jiayi, Yang Mohan, Wang Yanqi and Lai Yuchun were better than those of Wang Xuhang and Liu Ziyi, while the performances of Wu Jiayi and Lai Yuchun were the most prominent children, especially in oral communication.

The third stage of the experiment: the original control group family was changed into the experimental group, their overall situation was consistent with the original experimental group, and no significant difference in the participation of male and female parents was found. However, due to the lack of continuous supervision and guidance, the original experimental group's "participation" validity of the other five parents except Wu Jiayi showed different degrees of decline. The most obvious ones were Liu Ziyi and Wang Xuhang, and the learning validity of these two children has also decreased accordingly. In terms of the promotion mode of participation, parents most agree with the media mode, followed by the example mode and the help mode, and only parents who are good at oral English and who are more outgoing are willing to participate in the activity mode.

\section{CONCLUSION}

Experiments show that most non-English-teacher parents are willing and able to participate in family English education for young children, and the effective participation of parents can also stimulate the interest of young children in English learning and improve their learning. However, the improvement of parental participation level is inseparable from the guidance of experimental teachers and teachers of training institutions, and the ways in which the different categories of non-English-teacher parents participate in English education for young children should be different. The research team believes that parental participation should follow the principles of positive drive, multi-layer participation, different situation different solution, tangible display, multi-directional communication, resource sharing, etc., and various modes of participation such as media mode, example mode, and help mode should be used flexibly according to the different situations of parents. Based on the principle of convenience and validity control, the research team only selected 12 families as experiment subjects, and due to the limitations of experiment conditions, the experiment results obtained are inevitably biased, and the conclusions drawn from experience in the research conclusions are certainly quite a lot. Therefore, the research 
team believes that many issues need further study, such as: What kind of participation role do parents play in different age of children? What is the effective mode for raising the awareness and ability of parents to participate? What is the direction of guiding parents to participate in the development of effective resources? How to provide targeted guidance to different types of parents? What is the marginal point of participation behavior of different types of parents? How to construct a community promotion model for non-Englishteacher parents to participate in family English education for young children? What is the model for non-English-teacher parents to participate in family English education for children of all ages? What is the model for non-Englishteacher grandparents to participate in family English education for children of all ages? ... The research team believes that case studies and follow-up studies are needed in the future.

\section{REFERENCES}

[1] Yang Wen. English Teaching Method for Young Children [M]. China Book Press, 2010: 40-44. (in Chinese)

[2] Zhang Cuiyun. Research on the Design of English Teaching Activities for Young Children[D]. South China Normal University, 2007. (in Chinese)

[3] Feng Yi. Practical Research on Parents' Participation in Children's English Education[J]. Friends of Students (Primary School Edition), 2012(9): 49. (in Chinese). 